\title{
Aplicación del cuestionario de autoevaluación de la estrategia multimodal de la OMS para mejorar la práctica de higiene de manos en un hospital de tercer nivel
}

Application of the self-evaluation questionnaire of the WHO multimodal strategy to improve the practice of hand hygiene in a tertiary hospital

\footnotetext{
Alejandro de Arriba-Fernández ${ }^{1}$ (D) 0000-0001-5493-8780

Jesús Molina-Cabrillana²

Lluís Serra-Majem¹ (D) 0000-0002-9658-9061

1 Universidad de Las Palmas de Gran Canaria, Las Palmas de Gran Canaria, España.

${ }^{2}$ Complejo Hospitalario Universitario Insular de Gran Canaria, Servicio de Medicina Preventiva, Las

Palmas de Gran Canaria, España.
}

Fechas · Dates

Recibido: 2021.03 .06 Aceptado: 2021.08.26 Publicado: 2021.10.15

\section{Correspondencia $\cdot$ Corresponding Author}

Alejandro de Arriba-Fernández

alejandrodearribafdez@gmail.com

Universidad de Las Palmas de Gran Canaria. Paseo Blas Cabrera Felipe "Físico", 310, CP: 35016. Las Palmas Gran Canaria. 


\section{Resumen}

Objetivo. La higiene de manos (HM) es la medida individual más efectiva para reducir el riesgo de infecciones relacionadas con la atención sanitaria. Sin embargo, es habitual encontrar bajos resultados de cumplimiento de las indicaciones de HM entre profesionales sanitarios. El objetivo de este trabajo es evaluar la implementación de la aplicación de una estrategia multimodal para impulsar la promoción de la HM.

Métodos. Se utilizó la «Guía de aplicación de la estrategia multimodal de la Organización Mundial de la Salud para la mejora de la higiene de manos», conformado por: cambio del sistema, formación y entrenamiento, evaluación y retroalimentación, recordatorio en el lugar del trabajo, clima institucional de seguridad y vigilancia de la adherencia a la HM. La estrategia se planteó como un estudio de intervención para promover la HM en el Complejo Hospitalario Universitario Insular Materno Infantil en el período 2012-2020.

Resultados. El cumplimiento global de las indicaciones de $\mathrm{HM}$ al final del periodo de estudio fue del 59,2\%. El consumo de productos de base alcohólica aumentó con respecto a los años anteriores $(p<0,05)$, siendo en el último año de 70 litros por cada 1000 estancias. La encuesta reveló que la formación y la existencia de preparado de base alcohólica en cada punto de atención eran percibidas por parte de los profesionales sanitarios como los principales aspectos facilitadores de la práctica de HM.

Conclusiones. La implementación de una intervención multimodal mejoró significativamente la adhesión al lavado de manos y el uso de productos de base alcohólica entre los profesionales sanitarios. A pesar de tener formación previa sobre higiene de manos, los conocimientos de los profesionales son incompletos. La formación previa seguido del tiempo trabajado son los determinantes más importantes de las conocimientos y percepciones sobre higiene de manos.

Palabras clave: Lavado de manos; higiene de manos; adherencia; infección relacionada con la asistencia sanitaria; calidad; seguridad del paciente.

\section{Abstract}

Objective. Hand hygiene $(\mathrm{HH})$ is the single most important and effective measure to reduce the risk of healthcare-related infections. However, low compliance with $\mathrm{HH}$ indications among healthcare professionals is often low. The objective of this study was to evaluate the implementation of a multimodal strategy to promote $\mathrm{HH}$ among healthcare professionals.

Methods. We used the "Application guide of the multimodal strategy of the World Health Organization for the improvement of hand hygiene", which consists of: changes to the system, education and training, evaluation and feedback, workplace reminders, organizational safety climate and monitoring of compliance with $\mathrm{HH}$. The strategy was designed as an intervention study to promote $\mathrm{HH}$ in the Maternal and Child Insular University Hospital Complex in the period 2012-2020.

Results. Overall compliance with the $\mathrm{HH}$ indications at the end of the study period was $59.2 \%$. The use of alcoholic-based products increased with respect to previous years ( $p$ $<0.05$ ), reaching 70 liters/1000 hospital admissions in the final year. Healthcare professionals perceived training and accessibility to an alcohol-based preparation at each point of care as the most effective measures for promoting good $\mathrm{HH}$ practice. 
Conclusions. The implementation of a multimodal intervention significantly improved compliance with handwashing and the use of alcohol-based products among healthcare professionals. Despite having previous training on hand hygiene, $\mathrm{HH}$ knowledge among professionals remains incomplete. Prior training followed by time worked are the most important determinants of knowledge and perceptions about hand hygiene.

Keywords: Handwashing; hand hygiene; adherence; healthcare-related infection; quality; patient safety.

\section{Introducción}

La higiene de manos (HM), es decir, cualquier medida adoptada para la limpieza de las manos mediante fricción con un preparado de base alcohólica (PBA) o lavado con agua y jabón disminuye la transmisión cruzada de microorganismos a través de las manos de los profesionales sanitarios ${ }^{(1,2,3)}$ y ayuda a controlar las resistencias a los antibióticos ${ }^{(4)}$. Se considera, además, la medida más eficaz, económica y sencilla en la prevención de las infecciones relacionadas con la asistencia sanitaria (IRAS)(5). Sin embargo, la adhesión a los protocolos de HM entre los profesionales sanitarios no siempre es adecuada, siendo varias las barreras identificadas: irritación producida por los productos y/o la técnica de higiene, inaccesibilidad a los productos, prioridad en atender al paciente antes que en la antisepsia, uso de guantes en vez de antisepsia, olvido, desconocimiento de las recomendaciones, falta de tiempo, alta carga asistencial o falta de información respecto al impacto de la higiene en el control de las IRAS ${ }^{(1,6)}$. Esta falta de cumplimiento es un problema a nivel mundial(7).

La medición de la adherencia a la HM por parte de los profesionales es una práctica recomendada por la Organización Mundial de la Salud (OMS), en el contexto de la estrategia de mejora multimodal y debe ir acompañada de feedback a los profesionales para que tenga su mayor impacto ${ }^{(1,8)}$.

Las directrices de la OMS ponen de manifiesto que, prácticamente en todos los entornos, debería ser relativamente sencillo para los profesionales sanitarios comenzar a evaluar y mejorar la fiabilidad de las infraestructuras y las practicas de la HM. Este modelo anima a los profesionales sanitarios a lavarse las manos antes del contacto con el paciente, antes de realizar una tarea aséptica, después del riesgo de exposición a fluidos corporales, después del contacto con el paciente y después del contacto con el entorno del paciente ${ }^{(1)}$.

Para ello, la OMS desarrolló una estrategia de implementación multimodal y medidas para la $\mathrm{HM}^{9}$ que demostró su efectividad y adaptabilidad a diferentes entornos de atención médica con diferentes culturas y especificidades locales ${ }^{(10)}$.

El Servicio Canario de la Salud, con el fin de incrementar la seguridad de la atención sanitaria, está desarrollando políticas globales de seguridad de pacientes. Mejorar la adherencia a las recomendaciones de la HM por parte de los profesionales es una de las líneas estratégicas prioritarias. En este marco, en nuestro centro se 
está trabajando de forma activa en la mejora de la HM, siguiendo las directrices de organismos internacionales ${ }^{(1,11)}$.

Desde el Servicio de Medicina Preventiva del Complejo Hospitalario Universitario Insular Materno Infantil (CHUIMI) llevamos a cabo un programa para la vigilancia y el control de las IRAS en el que se incluyen actividades relacionadas con la promoción de la HM entre el personal sanitario desde el año 2012 ${ }^{(6,12)}$.

El principal objetivo de esta estrategia fue fomentar y promocionar el cumplimiento de la HM entre los profesionales sanitarios, voluntarios y familiares de los pacientes ingresados en el CHUIMI de forma mantenida en el tiempo y mejorar la seguridad de los pacientes utilizando para ello la estrategia multimodal de la OMS. El objetivo de este trabajo es evaluar su implementación.

\section{Métodos}

Diseño del estudio. Estudio evaluativo descriptivo y retrospectivo de una intervención multimodal para la mejora de la HM, que incluyó una intervención educativa en un grupo de profesionales, además de intervenciones organizativas.

Ámbito. El CHUIMI está formado por el Hospital Universitario Insular de Gran Canaria (HUIGC) y el Hospital Universitario Materno Infantil de Canarias (HUMIC), ambos centros de tercer nivel con 450 camas cada uno y acreditados para la docencia MIR.

Material. Se utilizó la «Guía de aplicación de la estrategia multimodal de la Organización Mundial de la Salud para la mejora de la higiene de manos», conformado por: cambio del sistema, formación y entrenamiento, evaluación y retroalimentación, recordatorio en el lugar del trabajo, clima institucional de seguridad y monitoreo de la adherencia a la HM manos ${ }^{(13)}$. El periodo de intervención se inició en el año 2012 y continuó hasta el año 2020.

\section{Estrategia multimodal de la OMS aplicada al CHUIMI}

1. Cambio del sistema con el propósito garantizar que se cuenta con la infraestructura necesaria para permitir a los profesionales sanitarios practicar la HM con PBA y, en la medida de los posible, en el punto de atención.

a) Infraestructura de HM. Todas las Unidades de hospitalización del CHUIMI cuentan con PBA dentro de la habitación. Las inspecciones de infraestructuras realizadas en 2020 han puesto de manifiesto que la mayoría de las habitaciones de las plantas de hospitalización disponen de dispensador de PBA y carteles informativos, de acuerdo con el objetivo consensuado del CHUIMI.

b) Estaciones de HM en el CHUIMI. Las estaciones de HM, ubicadas en las áreas asistenciales y en puntos estratégicos no asistenciales, son puntos dotados de mensajes informativos, PBA e información técnica. Tienen la misión de contribuir a incrementar la conciencia de realizar el acto de HM con PBA, tanto entre el per- 
sonal sanitario como entre los pacientes, visitas y familiares. Se comenzaron a implementar en el año 2012; actualmente el CHUIMI cuenta con más de 50 estaciones. Estas estaciones de HM están correctamente diseñadas y todas disponen de cartelería y dípticos informativos. Además, se encuentran censadas para facilitar su revisión y mantenimiento.

c) HM quirúrgica con PBA: El cambio de infraestructuras en quirófano para realizar HM quirúrgica (no convencional) con PBA comenzó a desarrollarse en 2013. Actualmente, todos los quirófanos cuentan con dicha infraestructura.

2. Formación y entrenamiento con el propósito de proporcionar formación con regularidad a todos los profesionales sanitarios sobre la importancia de la HM, basada en el modelo de "Los 5 momentos de la OMS"(1,13,14), mediante un programa formativo estructurado y evaluable.

a) Actividades formativas. Desde el año 2012 se está impartiendo un curso de HM avanzado ofertado para el personal del CHUIMI y que está acreditado por la Comisión Canaria de Acreditación de las Profesiones Sanitarias (1,3 créditos). Se usa metodología mixta on-line y presencial y requiere de la participación activa del alumno. Se incluye un taller sobre la técnica correcta usando luz ultravioleta y un videojuego de simulación.

- Talleres para voluntarios y familiares. Desde 2016 y hasta 2018 se ha incluido formación en HM a los grupos de voluntariado que realizan actividades en el CHUIMI, así como formación para padres y familiares de pacientes del Servicio de Oncohematología de HUMIC. Además, durante 2018 se ha realizado la formación de pacientes y familiares en aislamiento con el objetivo de incrementar la sensibilización y formación con medidas de aislamiento de contacto para que así puedan ejercer un papel más activo y responsable sobre su situación.

- Curso de HM quirúrgica con PBA en bloque quirúrgico: En 2016 se incluyó formación en HM con PBA para personal médico y de enfermería del bloque quirúrgico, necesario por la nueva dotación de PBA en los quirófanos.

- Talleres de prevención y control de infecciones con contenido de HM: Durante los años 2015 a 2018 se han impartido diversos talleres y sesiones clínicas con contenidos formativos en HM a diversos profesionales sanitarios, estudiantes de medicina y enfermería, internos residentes (enfermería y medicina) y formación profesional.

b) Mejorar disponibilidad de herramientas de formación, valorando documentos y herramientas a incluir en Intranet para apoyar la formación: Se revisa la bibliografía, artículos de interés y recomendaciones de organismos nacionales e internacionales.

3. Recordatorios en el lugar de trabajo con el propósito de garantizar la existencia y fácil accesibilidad de material para recordar a los profesionales sanitarios la importancia de la HM y las indicaciones y procedimientos adecuados para llevarla a cabo. 
a) Colocación y rotación de carteles en las áreas asistenciales. Entre 2012 y 2014 se desarrolló un proyecto específico con la elaboración de material promocional propio y novedoso, así como de las estaciones de HM.

Se realizaron varios formatos de material con un mensaje claro, sencillo y adaptado al lenguaje profesional y coloquial, con la idea de que el mensaje sobre la HM estuviera presente en todo el centro: Manteles desechables para la cafetería del centro, carteles con mensajes destinados tanto al profesional sanitario como a los usuarios del centro, carpetas, repartidas entre profesionales que acudieron a talleres formativos y dípticos para talleres y estaciones de $\mathrm{HM}$, calendarios con el 5 de mayo señalado en rojo y con mensaje de receta médica prescribiendo la HM. En relación con este nuevo material elaborado, en 2015 el CHUIMI consiguió el primer premio a la mejores prácticas clínicas seguras en el concurso de premios a "Implementación de buenas prácticas en seguridad del paciente", promovido por la Agencia de Calidad del Ministerio de Sanidad, Servicios Sociales e Igualdad en el contexto de la estrategia europea "Patient Safety and Quality of Care".

Por otra parte, tras la revisión de infraestructuras de HM, las plantas de hospitalización están dotadas de cartelería de las distintas campañas de HM implementadas por el Ministerio de Sanidad, por el Servicio Canario de la Salud y las propias del CHUIMI.

b) Revisión y mantenimiento de la Intranet. Los documentos y herramientas de HM que se consideran con un contenido apropiado para Intranet se actualizan periódicamente, aunque no existe un programa de actividades definido propiamente dicho.

4. Evaluación y retroalimentación con el propósito de inspeccionar las infraestructuras y las prácticas de HM, junto con evaluación de conocimientos y/o percepción por parte de los profesionales sanitarios.

a) Revisión y mantenimiento continuo de la presencia y el estado del material promocional (estaciones HM, puntos de atención, salas comunes de reuniones, etc.) Se realizan valoraciones periódicas anuales de la infraestructura de HM en las unidades de hospitalización, urgencias, hospital de día y de las estaciones de HM, emitiéndose un informe anual.

b) Evaluación del conocimiento y percepción de los trabajadores. En el año 2020 se realizó un estudio transversal y analítico a través de un cuestionario auto administrado propuesto por la OMS ${ }^{(15,16)}$ a un colectivo de 170 profesionales sanitarios. Se calculó la asociación entre las características de los profesionales y el conocimiento y la percepción sobre la HM mediante una regresión logística. Por otra parte, el mismo cuestionario se auto administrado a un colectivo de 35 directivos y mandos intermedios ${ }^{(17)}$.

c) Monitorización semestral del consumo de PBA. El consumo de PBA se centra en las unidades de hospitalización y se expresa en litros por cada 1.000 estancias. Para la construcción del indicador se excluyen los litros entregados en consultas externas, Psiquiatría, Urgencias, hospital de día, área quirúrgica y otros servicios donde el paciente no permanezca ingresado. 
d) Monitorización de la adherencia a la HM. Es una actividad que se ha venido realizando dos veces al año en los Servicios de urgencias de adultos y de pediatría y en las Unidades de Cuidados Intensivos, tanto de pediatría como de adultos, y en la Unidad de Cuidados Intensivos Neonatal. En 2020 se ha continuado la observación de la adherencia en todo el CHUIMI y observando los 5 momentos, de acuerdo con las recomendaciones de la OMS1.

5. Clima institucional de seguridad con el propósito de crear un entorno que propicie la sensibilización sobre las cuestiones de seguridad del paciente y garantizar al mismo tiempo que la mejora de la HM se considere una prioridad a todos los niveles de la institución.

a) Actividades 5 de mayo. El 5 de mayo, Jornada Mundial de la HM a iniciativa de la OMS, se celebra en el Complejo desde el año 2009. Estas jornadas anuales han consistido en la organización e implementación de una serie de actividades de sensibilización sobre la importancia de la HM (sesiones formativas y de difusión, redacción y difusión de notas divulgativas, sesión clínica general con la asistencia del Equipo Directivo, reparto de material promocional, promoción de la HM en hall de entrada, mesa informativa con material informativo, mensajes sobre la $\mathrm{HM}$ al encender el ordenador, noticia en pagina web, diapositivas alusivas a la HM en todas las actividades formativas realizadas, actividades con voluntariado en plantas de hospitalización pediátrica, talleres de dibujo, manualidades y/o musicales con los niños ingresados con mensaje de HM y proyección de vídeos en hall de entrada, musicoterapia, y "photocall"). Se contó con la colaboración de la Unidad de Calidad y Formación Continuada, Pediatría, Mantenimiento, Servicio de Informática, Departamento de Comunicaciones, Gabinete de prensa y el equipo directivo.

b) Comité de Higiene de Manos. En 2016 se crea el Comité de HM con el propósito de contribuir a incrementar el clima de seguridad institucional en relación con la HM. Los objetivos específicos son: conocer el plan de actuación del CHUIMI y participar de su evaluación; identificar factores facilitadores y barreras para la mejora, aportando sugerencias de actividades; conocer y difundir los logros alcanzados; fomentar la participación de todos los profesionales sanitarios en las actividades del 5 de mayo; promover actividades de participación del paciente-familia. El comité está compuesto por profesionales de diversos Servicios y Unidades con implicación en los programas de mejora relacionados con la HM, como son la Comisión de Infecciones y Seguridad del Paciente así como representantes del Equipo Directivo (1 de la división médica y 3 de la de enfermería).

c) Autoevaluación del programa. La OMS pone a disposición de los centros una herramienta de autoevaluación elaborada por consenso de un grupo de expertos y que valora lo que se considera los puntos esenciales de los programas de mejora de la HM en centros sanitarios ${ }^{(18)}$.

\section{Análisis estadístico}

Se diseñó una hoja de recogida de datos al efecto y la información se registró en una base de datos normalizada. El indicador de cumplimiento con la HM se cal- 
culó como la proporción de oportunidades definidas por los cinco momentos (antes del contacto con el paciente, antes de tareas asépticas, después de riesgo de exposición a líquidos corporales, después del contacto con el paciente y después del contacto con su entorno), en las que realizaron acciones de lavado con agua y jabón o fricción de manos con un desinfectante alcohólico. La comparación de estas proporciones del "antes" y el "después" se hizo por medio de la prueba exacta de Fisher ante la imposibilidad de corrección por la no independencia de las observaciones, dado que un mismo trabajador podía ser observado en más de una oportunidad. El tratamiento estadístico de los datos se ha realizado mediante la aplicación estadística Statistical Package for the Social Sciences, v 24 y Microsoftß Excel (2010).

\section{Resultados}

En el año 2020 el complejo ya disponía de dispensadores de PBA en el punto de atención al paciente en el 100\% de las camas.

Se han realizado entre 7 y 11 ediciones por año del curso de HM avanzado. Actualmente se han realizado 64 ediciones con 924 participantes en total. Lo más destacable es la participación de los profesionales, tal como se puede ver en la Tabla 1. La mayor participación ha correspondido al personal de enfermería (colectivo más numeroso y con mayores contactos con el paciente), lo que ha estado motivado además porque en 2015 la Dirección de Enfermería incluyó este curso entre los objetivos ligados a incentivos en diversa áreas, lo que supuso un incremento importante de asistentes. Esta fue una de las intervenciones más bien valoradas por los profesionales para promover el conocimiento y cumplimiento de las indicaciones de HM (Tabla 2).

Tabla 1: Resumen de la actividad del curso de higiene de manos avanzado en el CHUIMI (Hospital Universitario Insular de Gran Canaria y el Hospital Universitario Materno Infantil de Canarias).

Tabla 1. Resumen de la actividad del curso de higiene de manos avanzado en el CHUIMI

\begin{tabular}{|l|c|c|c|c|c|c|c|c|c|}
\hline & $\mathbf{2 0 1 2}$ & $\mathbf{2 0 1 3}$ & $\mathbf{2 0 1 4}$ & $\mathbf{2 0 1 5}$ & $\mathbf{2 0 1 6}$ & $\mathbf{2 0 1 7}$ & $\mathbf{2 0 1 8}$ & $\mathbf{2 0 1 9}$ & Total \\
\hline № ediciones & 7 & 9 & 7 & 11 & 10 & 11 & 9 & 8 & 64 \\
\hline № asistentes & 93 & 72 & 45 & 288 & 132 & 190 & 104 & 130 & 924 \\
\hline Ratio (asist./edición) & $\mathbf{1 3 , 3}$ & $\mathbf{8 , 0}$ & $\mathbf{6 , 4}$ & $\mathbf{2 6 , 2}$ & $\mathbf{1 3 , 2}$ & $\mathbf{1 7 , 3}$ & $\mathbf{1 1 , 6}$ & $\mathbf{1 6 , 3}$ & $\mathbf{1 4 , 4}$ \\
\hline Enfermeros & 38 & 22 & 10 & 250 & 36 & 97 & 41 & 36 & 494 \\
\hline Auxiliares & 28 & 42 & 29 & 1 & 61 & 62 & 44 & 60 & 267 \\
\hline Médicos & 3 & 3 & 4 & 37 & 32 & 26 & 14 & 7 & 119 \\
\hline Otros & 12 & 5 & 2 & 0 & 3 & 5 & 5 & 27 & 32 \\
\hline
\end{tabular}


Tabla 2: Valoración media de la actividad formativa del curso de higiene de manos avanzado.

Organización y metodología de la actividad formativa $\quad 8,5$

Evaluación global de la actividad Formativa $\quad 8,9$

Evaluación global de la unidad de formación continuada y docencia $\quad 8,7$

Evaluación global al profesor 9,2

La adherencia total a la HM ha aumentado del $42,5 \%$ en 2018 , al $47,6 \%$ en 2019 y al $59,2 \%$ en $2020(p<0,05)$. La adherencia total fue mayor en los momentos después del contacto con el paciente (67\%) que en los momentos anteriores al contacto (48\%) (Tabla 3). El área con mayor adherencia fue diálisis (83\%). Se distingue una mayor adherencia en áreas abiertas que en áreas de hospitalización (65\% vs 56\%). Se determinó una mayor adherencia en facultativos (73\%) y enfermeras (74\%), que en auxiliares de enfermería $(50 \%)(p<0,05)$. Se observó una tendencia al aumento del consumo de PBA en las áreas quirúrgicas $(p<0,05)$.

Tabla 3: Adherencia por categoría y tipo de áreas, según momentos antes y después del contacto.

\begin{tabular}{|c|c|c|c|c|c|c|c|c|c|}
\hline & \multicolumn{3}{|c|}{ HUIGC 2020} & \multicolumn{3}{|c|}{ HUIGC 2019} & \multicolumn{3}{|c|}{ HUIGC 2018} \\
\hline & $\begin{array}{l}\text { Adher. } \\
\text { ANTES }\end{array}$ & $\begin{array}{l}\text { Adher. } \\
\text { DESPUÉS }\end{array}$ & $\begin{array}{l}\text { Adher. } \\
\text { TOTAL }\end{array}$ & $\begin{array}{l}\text { Adher. } \\
\text { ANTES }\end{array}$ & $\begin{array}{l}\text { Adher. } \\
\text { DESPUÉS }\end{array}$ & $\begin{array}{l}\text { Adher. } \\
\text { TOTAL }\end{array}$ & $\begin{array}{l}\text { Adher. } \\
\text { ANTES }\end{array}$ & $\begin{array}{l}\text { Adher. } \\
\text { DESPUÉS }\end{array}$ & $\begin{array}{l}\text { Adher. } \\
\text { TOTAL }\end{array}$ \\
\hline & $\mathrm{N}(\%)$ & $\mathrm{N}(\%)$ & $\mathrm{N}(\%)$ & $\mathrm{N}(\%)$ & $\mathrm{N}(\%)$ & $\mathrm{N}(\%)$ & $\mathrm{N}(\%)$ & $\mathrm{N}(\%)$ & $\mathrm{N}(\%)$ \\
\hline Total & $\begin{array}{c}329 \\
(48,1)\end{array}$ & $412(67,3)$ & $\begin{array}{c}741 \\
(59,2)\end{array}$ & $\begin{array}{c}295 \\
(36,9)\end{array}$ & $428(54,9)$ & $\begin{array}{c}723 \\
(47,6)\end{array}$ & $\begin{array}{c}1512 \\
(27,3)\end{array}$ & $\begin{array}{r}1902 \\
(54,6)\end{array}$ & $\begin{array}{c}3414 \\
(42,5)\end{array}$ \\
\hline \multicolumn{10}{|c|}{ Por Categoría } \\
\hline Enfermería & $\begin{array}{l}167 \\
(52)\end{array}$ & $186(73,5)$ & $\begin{array}{c}353 \\
(63,9)\end{array}$ & $\begin{array}{c}157 \\
(60,6)\end{array}$ & $189(57,7)$ & $\begin{array}{c}346 \\
(50,6)\end{array}$ & $681(37,7)$ & $883(66,6)$ & $\begin{array}{c}1564 \\
(54,0)\end{array}$ \\
\hline Auxiliares & $\begin{array}{c}72 \\
(31,6)\end{array}$ & $113(50,4)$ & $\begin{array}{c}185 \\
(43,2) \\
\end{array}$ & $\begin{array}{c}80 \\
(27,5)\end{array}$ & $140(40,7)$ & $\begin{array}{c}220 \\
(35,9)\end{array}$ & $396(15,7)$ & $551(47,2)$ & $\begin{array}{c}947 \\
(34,0)\end{array}$ \\
\hline Facultativos & $\begin{array}{c}76 \\
(57,5)\end{array}$ & $98(72,6)$ & $\begin{array}{c}174 \\
(66,3)\end{array}$ & $\begin{array}{c}48 \\
(39,6)\end{array}$ & $84(75)$ & $\begin{array}{c}132 \\
(62,1)\end{array}$ & $336(21,7)$ & $368(41,8)$ & $\begin{array}{c}704 \\
(32,2)\end{array}$ \\
\hline
\end{tabular}

N: número de observaciones; \%: tasa de adherencia

Hospital Universitario Insular de Gran Canaria : HUIGC

Tabla 4: Adherencia global y uso de productos de base alcohólica (PBA) por áreas asistenciales

\begin{tabular}{|c|c|c|c|c|c|c|c|c|c|}
\hline & \multicolumn{3}{|c|}{ Enero-Diciembre, 2020} & \multicolumn{3}{|c|}{ Enero-Diciembre, 2019} & \multicolumn{3}{|c|}{ Enero-Diciembre, 2018} \\
\hline & $\begin{array}{c}\mathrm{N}^{\circ} \text { oportu- } \\
\text { nidades }\end{array}$ & $\begin{array}{l}\% \text { Adhe- } \\
\text { rencia }\end{array}$ & $\begin{array}{l}\% \text { Uso } \\
\text { PBA }^{a}\end{array}$ & $\begin{array}{l}\mathrm{N}^{\circ} \text { oportu- } \\
\text { nidades }\end{array}$ & $\begin{array}{c}\% \\
\text { Adhe- } \\
\text { rencia }\end{array}$ & $\begin{array}{c}\% \\
\text { Uso } \\
\text { PBA }^{a}\end{array}$ & $\begin{array}{l}\mathrm{N}^{\circ} \text { oportu- } \\
\text { nidades }\end{array}$ & $\begin{array}{l}\text { \% Adhe- } \\
\text { rencia }\end{array}$ & $\begin{array}{l}\% \text { Uso } \\
\text { PBA }^{a}\end{array}$ \\
\hline Total HUIGC & 741 & 59,2 & 54,6 & 711 & 47,6 & 42,9 & 3108 & 42,5 & 32,1 \\
\hline
\end{tabular}

a Se calcula como el número de veces que se usa PBA x 100 / total de oportunidades observadas de HM

La encuesta a profesionales sanitarios determinó que la mayoría recibió formación en HM 120 (70,6\%) y prefería utilizar PBA 105 (61,8\%). Además, consideraron las manos como principal vía de transmisión cruzada de microorganismos $(81,3 \%)$. Respecto a las barreras para no realizar HM, la más mencionada fue el daño que produce en las manos (42,1\%). La medida considerada más efectiva 
para aumentar el cumplimiento de HM fue la presencia de instrucciones claras y visibles en todas las áreas de trabajo (78\%).

La totalidad de los encuestados consideraron alta o muy alta la repercusión de las IRAS en los gastos sanitarios y en el desenlace de la enfermedad del paciente. Además, percibían que la eficacia de la HM en la prevención de las IRAS es alta o muy alta.

La mayoría sobrestimó el porcentaje de pacientes hospitalizados que contrae una IRAS y la adherencia a la HM de los trabajadores del centro. Las medidas consideras prioritarias para mejorar la HM fueron que exista preparado de base alcohólica en cada punto de atención y que los profesionales sanitarios reciban formación sobre la HM (94,3\%). La formación previa seguido del tiempo trabajado son los determinantes más importantes de las conocimientos y percepciones sobre HM.

A pesar de tener experiencia previa en programas de HM, las percepciones de los directivos son en ocasiones sesgadas, sobrestimando la adherencia a la HM y las IRAS.

El comité de HM ha mantenido un ritmo constante de trabajo, con unas 10 reuniones al año y un porcentaje de asistencia de los miembros superior al 70\%.

La herramienta de evaluación de la OMS determinó que desde sus inicios el programa parecía encontrarse en el nivel intermedio (> 250 puntos) (Figura 1). Se observó un descenso en el nivel de desarrollo hasta 2016, año a partir del cual empieza a detectarse nuevamente una mejora, de forma que en estos momentos estamos de nuevo en el nivel intermedio.

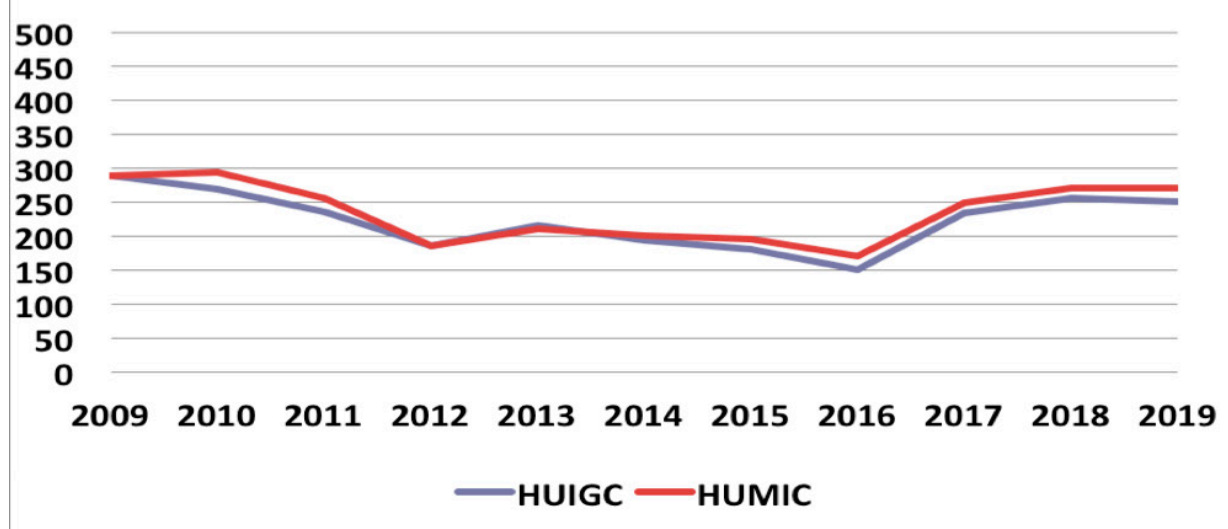

Figura 1: Evolución del nivel de desarrollo del programa de higiene de manos en el CHUIMI (Hospital Universitario Insular de Gran Canaria y el Hospital Universitario Materno Infantil de Canarias) (20092019).

Como se observa en la Figura 2, el consumo de PBA en el CHUIMI ha aumentado desde 2012, destacando el marcado incremento a partir de $2017(p<0,05)$. 


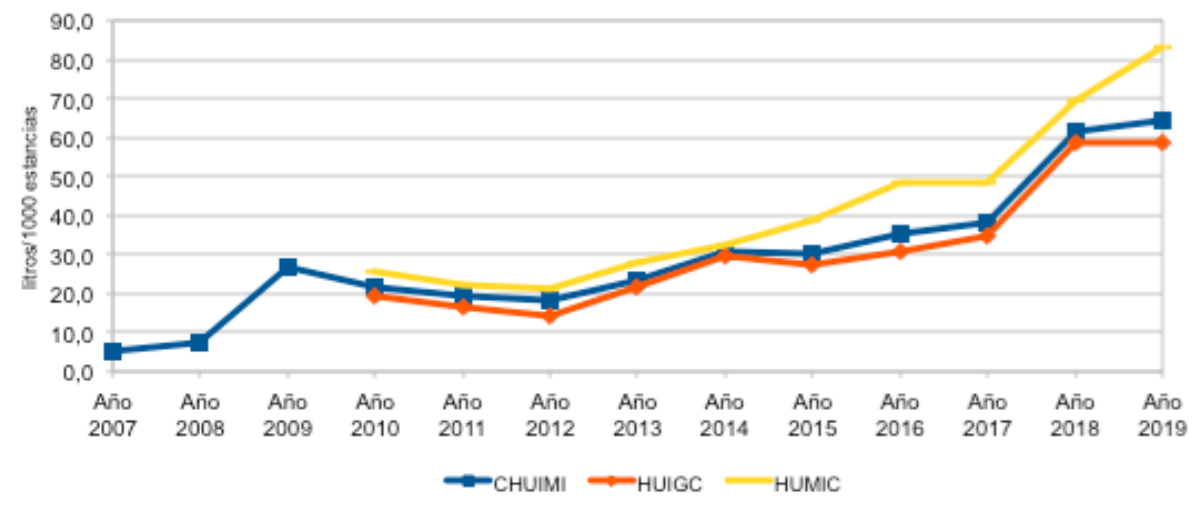

Figura 2: Consumo de productos de base alcohólica en el CHUIMI (Hospital Universitario Insular de Gran Canaria y el Hospital Universitario Materno Infantil de Canarias)en el periodo 2007-2019 (Iitros/1000 días de estancia).

En el 92,9\% de las áreas de hospitalización hay cartelería sobre la técnica de la HM. En el 2020 se detectó una mayor disponibilidad de material informativo que años previos en las unidades de hospitalización.

\section{Discusión}

Se ha puesto de manifiesto una mejora en las prácticas de $\mathrm{HM}$ al final del periodo de estudio entre los profesionales sanitarios del CHUIMI, suponemos que el aumento en la adherencia está asociado con la implantación de la estrategia multimodal de la OMS.

Este y otros estudios recientes demuestran que implementar una estrategia multimodal para la HM aumenta significativamente el cumplimiento de esta medida, independientemente del tipo de trabajador de salud o departamento hospitalario(10,19-24). Sin embargo, no siempre fue así. Algunos estudios concluyeron que la intervención no afecta el cumplimiento de la $\mathrm{HM}^{(25,26)}$.

El índice de cumplimiento de HM varía en todo el mundo, indicando numerosos estudios internacionales que se encuentra por debajo del 50\%(27,28). En España se han publicado varios artículos sobre el cumplimiento de la HM en hospitales de distintas comunidades autónomas donde los datos de adherencia se encuentran alrededor del $40 \%$, aunque varían ${ }^{(29-31)}$. La adhesión evaluada en nuestro estudio tras puesta en marcha de la estrategia multimodal superó la aportada por Rodriguez-Villar (44,3\%) en 2018(29) y Sanchez-Paya en $2007^{(31)}$ (30\%) y fue similar a la aportada por Arredondo-Provecho en 2019 (57,8\%) $)^{(30)}$.

Los indicadores analizados, así como la revisión de infraestructuras parecen mostrar una mejoría en el programa de HM en nuestro Complejo. La herramienta de evaluación de la OMS determinó que desde sus inicios el programa de HM en el CHUIMI se encontraba en el nivel intermedio, tal vez debido a una serie de forta- 
lezas y oportunidades, como mayor disponibilidad de recursos, mayor impulso e implicación a nivel institucional (autonómico y central). Sin embargo, se observó un descenso en el nivel de desarrollo hasta 2016, año a partir del cual empieza a detectarse nuevamente una mejora, de forma que en estos momentos estamos de nuevo en el nivel intermedio.Esta herramienta proporciona una oportunidad para reflexionar sobre los recursos existentes y los logros alcanzados, ayudando a focalizar los aspectos que es necesario mejorar. Consideramos necesario reorientar y redefinir algunos de sus principales componentes para seguir mejorando hasta conseguir la excelencia y liderazgo.

La existencia de los talleres avanzados de HM se consideró imprescindible y necesario para favorecer y mantener una formación continuada del personal sanitario en prevención y control de infecciones. La promoción de la asistencia a esta actividad no supone un incremento del gasto, más bien al contrario, sería una manera de incrementar la eficiencia ${ }^{5}$. La percepción de los responsables de esta actividad es que la formación en HM fue bastante positiva; y por lo general, la mayor parte de los trabajadores que han participado se mostraron interesados y su valoración fue bastante satisfactoria, llegando a comentar que antes no se habían planteado la importancia de hacer una buena HM.

Las actividades del 5 de mayo son actuaciones que han requerido una preparación intensa previa, al tratarse de trabajos creativos, manuales y que han requerido coordinación con otros profesionales (profesores, musicoterapeutas, caricaturista, ilustrador, imprenta). Se trata de una actividad primordial pero que requiere de gran inversión de tiempo y esfuerzo, además de la motivación necesaria para que las actividades sean novedosas y que puedan causar impacto en los profesionales del CHUIMI.

Desde el punto de vista económico y logístico, el apoyo de los líderes de la institución fue de gran importancia. El compromiso y apoyo de los médicos jefes de servicio y supervisoras del área de enfermería de las distintas unidades hizo que la campaña de HM tuviese el efecto esperado, entregándole un sentido positivo a esta importante práctica. Creemos que los mayores avances se conseguirán manteniendo estas actividades a medio y largo plazo.

En conclusión, la implementación de una intervención multimodal mejoró significativamente la adhesión al lavado de manos entre los profesionales sanitarios y aumentó el consumo de PBA en el Hospital. Consideramos que, a pesar de su enorme importancia en la prevención de la transmisión de enfermedades, la práctica correcta de la HM no ha sido suficientemente estudiada. La investigación debería centrarse en cómo mantener una alta adherencia a la HM después de la pandemia de COVID-19.

\section{Agradecimientos}

A todo el equipo de médicos, enfermeras y residentes del Servicio de Medicina Preventiva del CHUIMI por su por su implicación, apoyo y participación en la recogida de datos, sin los cuales este trabajo no hubiera sido posible. 


\section{Financiación}

Los autores declaran que no han recibido financiación para la realización de este estudio.

\section{Conflicto de intereses}

Los autores declaran no tener conflictos de intereses.

\section{Bibliografía}

1. World Health Organization. WHO Guidelines on Hand Hygiene in Health Care: First Global Patient Safety Challenge Clean Care Is Safer Care. Geneva: World Health Organization; 2009.

2. Allegranzi $B$, Pittet $D$. Role of hand hygiene in healthcare-associated infection prevention. J Hosp Infect. 2009;73(4):305-15. doi: 10.1016/j.jhin.2009.04.019.

3. Merino-Plaza MJ, Rodrigo-Bartual V, Boza-Cervilla M, García-Llopis A, Gomez-Pajares F, Carrera-Hueso FJ, Fikri-Benbrahim N. ¿Cómo incrementar la adhesión del personal sanitario al protocolo de higiene de manos?. Rev Esp Salud Pública. 2018; 92: e201810072.

4. Kingston L, O'Connell NH, Dunne CP. Hand hygiene-related clinical trials reported since 2010: a systematic review. J Hosp Infect. 2016;92(4):309-320. doi: 10.1016/j. jhin.2015.11.012.

5. Händehygiene in Einrichtungen des Gesundheitswesens : Empfehlung der Kommission für Krankenhaushygiene und Infektionsprävention (KRINKO) beim Robert Koch-Institut (RKI). Bundesgesundheitsblatt Gesundheitsforschung Gesundheitsschutz. 2016;59(9):1189-220. doi: 10.1007/s00103-016-2416-6.

6. Molina-Cabrillana J, Dorta-Hung ME, Otero Sanz L, Henández Vera JR, Martín-Rodríguez MM, García de Carlos P. Influencia del material promocional sobre higiene de manos en la cultura de seguridad de un hospital de tercer nivel. Rev Calid Asist. 2016;31 Suppl 1:55-61. doi: 10.1016/j.cali.2016.01.002

7. Stewardson AJ, Sax H, Gayet-Ageron A, Touveneau S, Longtin Y, Zingg W, Pittet

D. Enhanced performance feedback and patient participation to improve hand hygiene compliance of health-care workers in the setting of established multimodal promotion: a single-centre, cluster randomised controlled trial. Lancet Infect Dis. 2016;16(12):1345-1355. doi: 10.1016/S1473-3099(16)30256-0.

8. Sax H, Allegranzi B, Chraïti MN, Boyce J, Larson E, Pittet D. The World Health Organization hand hygiene observation method. Am J Infect Control. 2009;37(10):82734. doi: 10.1016/j.ajic.2009.07.003. 
9. Pittet D, Allegranzi B, Storr J. The WHO Clean Care is Safer Care programme: field-testing to enhance sustainability and spread of hand hygiene improvements. J Infect Public Health. 2008;1(1):4-10. doi: 10.1016/j.jiph.2008.08.006.

10. Ben Fredj S, Ben Cheikh A, Bhiri S, Ghali H, Khefacha S, Dhidah L, Merzougui L, Ben Rejeb M, Said Latiri H. Multimodal intervention program to improve hand hygiene compliance: effectiveness and challenges. J Egypt Public Health Assoc. 2020;95(1):11. doi: 10.1186/s42506-020-00039-w.

11. Boyce JM, Pittet D. Guideline for Hand Hygiene in Health-Care Settings: Recommendations of the Healthcare Infection Control Practices Advisory Committee and the HICPAC/SHEA/APIC/IDSA Hand Hygiene Task Force. U.S.A.: Centers for Disease Control and Prevention; 2002.Report No.: MMWR 2002;51(RR-16). Disponible en: http://www.cdc.gov/mmwr/preview/mmwrhtml/rr5116a1.htm

12. Molina-Cabrillana J, Alvarez-León EE, Quori A, García-de Carlos P, López-Carrió I, Bolaños-Rivero M, Hernández-Vera JR, Ojeda-García I, Córdoba-Tasi E, Ramírez-Rodríguez A, Henríquez-Ojeda A. Impacto de la mejora de la higiene de las manos sobre las infecciones hospitalarias. Rev Calid Asist. 2010;25(4):215-22. doi: 10.1016/j.cali.2010.02.002.

13. Wold Health Organization. A guide to the implementation of the WHO multimodal hand hygiene improvement strategy. Geneva: WHO; 2009 [citado 21 Nov 2020]. Disponible en: http://www.who.int/gpsc/5may/Guide_to_Implementation.pdf.

14. Multimodal intervention to increase hand hygiene compliance. Description of safe clinical practice (SPC) and implementation process for Work Package 5. PaSQ Project [consultado 21 Nov 2020]. Disponible en: http://www.pasq.eu/Wiki/ SCP/WorkPackage5ToolBoxes/HandHygiene.aspx

15. Organización Mundial de la Salud. Cuestionario de percepción sobre la higiene de las manos destinado a los profesionales sanitarios. 2010 [citado 4 Nov 2020]. Disponible en: https://www.mscbs.gob.es/organizacion/sns/planCalidadSNS/ docs/cuestionario_percepcion_prof_oms.pdf

16. Organización Mundial de la Salud. Cuestionario acerca de los conocimientos sobre la higiene de las manos destinado a los profesionales sanitarios. 2010 [citado 4 Nov 2020]. Disponible en: https://www.mscbs.gob.es/organizacion/sns/ planCalidadSNS/docs/cuestionario_conocimientos_prof_oms.pdf

17. Organización Mundial de la Salud. Cuestionario de percepción sobre la higiene de las manos destinado directivos. 2010 [citado 4 Nov 2020]. Disponible en https://www.mscbs.gob.es/organizacion/sns/planCalidadSNS/docs/cuestionario_percepcion_directivos_oms.pdf

18. World Health Organization. Hand Hygiene Self-Assessment Framework. 2010 [citado 8 Nov 2020]. Disponible en: http://www.who.int/gpsc/country_work/hhsa_ framework/en/index.html 
19. Restrepo AV, Valderrama MP, Correa AL, Mazo LM, González NE, Jaimes F. Implementación de la estrategia "Atención Limpia es Atención Segura" en un hospital de tercer nivel en Medellín, Colombia. Rev chil infectol. 2014:31(3):280-6.

20. Fariñas-Alvarez C, Portal-María T, Flor-Morales V, Aja-Herrero A, Fabo-Navarro M, Lanza-Marín S, et al. Estrategia multimodal para la mejora de la adherencia a la higiene de manos en un hospital universitario. Rev Calid Asist. 2017;32(1):50-56.

21. Sobrequés J, Espuñes J, Bañeres J. Estrategia para mejorar la práctica de higiene de manos en Catalunya. Med Clin (Barc). 2014;143(Supl 1):36-42.

22. Trejo-González R, Yacaman-Handal RE, Hernández-González LA, Soto-López ME, Gabriela-Koretzky S. Higiene de manos 90/90: programa de mejora continua para la adherencia a la higiene de manos. An Med (Mex). 2019;64(3):190-195.

23. Batista J, Silva DP, Nazário SS, Cruz EDA. Multimodal strategy for hand hygiene in field hospitals of COVID-19. Rev Bras Enferm. 2020;73(Suppl 6):e20200487.

24. Fuentes-Ferrer ME, Peláez-Ros B, Andrade-Lobato R, del Prado-González N, Cano- Escudero S, Fereres-Castiel J. Efectividad de una intervención para la mejora del cumplimiento en la higiene de manos en un hospital de tercer nivel. Rev Calid Asist. 2012;27(1):3-10.

25. Muto CA, Sistrom MG, Farr BM. Hand hygiene rates unaffected by installation of dispensers of a rapidly acting hand antiseptic. Am J Infect Control. 2000;28(3):273-6.

26. Dorsey ST, Cydulka RK, Emerman CL. Is handwashing teachable?: failure to improve handwashing behavior in an urban emergency department. Acad Emerg Med Off J Soc Acad Emerg Med. 1996;3(4):360-5.

27. Pan A, Domenighini F, Signorini L, Assini R, Catenazzi P, Lorenzotti S, et al. Adherence to hand hygiene in an Italian long-term care facility. Am J Infect Control. 2008;36(7):495-7.

28. Albright J, White B, Pedersen $D$, Carlson P, Yost L, Littau C. Use patterns and frequency of hand hygiene in healthcare facilities: Analysis of electronic surveillance data. Am J Infect Control. 2018;46:1104-9.

29. Rodríguez-Villar D, Del-Moral-Luque JA, San-Román-Montero J, Gil-de-Miguel Á, Rodríguez-Caravaca G, Durán-Poveda M. Adherencia a la higiene de manos con soluciones hidroalcohólicas en estudiantes de medicina. Estudio descriptivo transversal. Rev Esp Quimioter. 2019;32(3):232-7.

30. Arredondo-Provecho AB, Horcajo-Díaz E, Cerrillo-González I, Morato-Cerro VM, Pérez-Ortiz M, Rodríguez-Caravaca G. Evolución de la adherencia a la higiene de manos en un hospital de la Comunidad de Madrid. Rev Esp Salud Pública. 2020;94(1):e202007075.

31. Sánchez-Payá J, Galicia-García MD, Gracia-Rodríguez RM, García-González C, Fuster-Pérez M, López-Fresneña N, et al. Grado de cumplimiento y determinantes de las recomendaciones sobre la higiene de manos. Enferm Infecc Microbiol Clin. 2007;25(6):369-75. 